
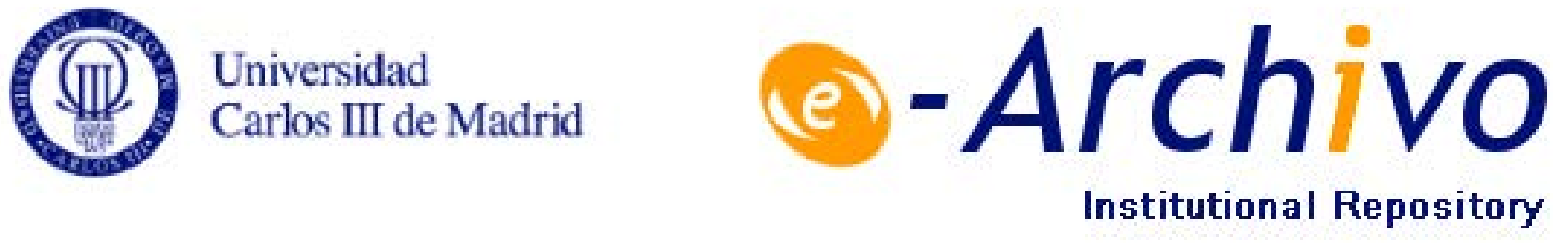

This is a postprint version of the following published document:

Gutiérrez, R., Ramírez, M., Olmeda, E. and V. Díaz. Practical Case Application for Stress Model Validation and Enhancement by Means of Metrological Tools. Strain (2015) v. 51, 5, 379-390.

DOI: $10.1111 /$ str.12149

(C) 2015 Wiley Publishing Ltd. 


\title{
Practical Case Application for Stress Model Validation and Enhancement by Means of Metrological Tools
}

\author{
R. Gutiérrez, M. Ramírez, E. Olmeda and V. Díaz \\ Mechanical Engineering Department, Universidad Carlos III de Madrid, Madrid, Spain \\ E-mail: ragutier@ing.uc3m.es
}

\begin{abstract}
Models consider ideal and simplified situations that will never be met in the real case. The process of comparing model predictions and experimental observation is in the basis of scientific research. This comparison is however complicated because of the uncertainties of the model input data and the difficulty to control the accuracy of the tests and to obtain a significant statistical sampling. Moreover, there isn't yet a consensus on a validation parameter. This paper presents a three-step validation procedure that allows quantifying the application limits of a two-dimensional stress model in a three-dimensional situation. A global uncertainty model is calculated comprising the uncertainty of the model and also the uncertainty coming from the experimental results. The $E_{N}$ number, a statistical magnitude for interlaboratory comparisons, is used to analyse the compatibility between the experimental and theoretical results. Finally, a bootstrapping method is proposed to calculate the coverage interval of the sampling and determine if new experiments should be carried out. Numerical results of this new validation procedure are provided for the case under study. It is also demonstrated that the computed uncertainty budget can be a useful tool to enhance the two-dimensional model by enlarging its uncertainty limits.
\end{abstract}

KEY WORDS: model validation, strain gauge measurement, stress concentration factor, uncertainty analysis

\section{Introduction}

Deterministic structural mechanics has been extensively used by mechanical engineers as they needed models that could predict the behaviour of the designed structures and components [1]. Nowadays, a more realistic analysis and elegant design of structures can be performed, if the uncertainty due to the random behaviour of the model's parameters is taken into account [1-3]. Many are the benefits of considering the uncertainties of the parameters of the model. On the one hand, the model can provide a range of probabilities and thus, more realistic values, and additional uncertainty components can be added when the ideal conditions of the model are not met. On the other hand, once the effects of the uncertainties of the parameters are quantified, it is possible to improve the results by acting on the accuracy of those parameters showing a higher influence in the final result [4].

Moreover, model verification and validation has to consider the uncertainty in the model and the experimental results [5], as the quantitative comparisons of model predictions and experiments are incomplete, only if the difference between both is calculated [6]. The traditional graphical comparison is also subjective and depends on the graph scale [7].

Therefore, if the validation methodology of an engineering model is based on the acceptance of a certain error, it should also include the uncertainty inherent to it [4].

Validation procedures have revealed to be complex, so engineering associations and research centres have published guides for a better understanding of the process
$[3,4,8]$, even though the approach has not yet been unified [6]. The reviewed literature evidences that the publication of practical cases in the field of stress model validation is scarce. This paper presents a three-step validation procedure (in advance TSVP) that allows quantifying the application limits of a two-dimensional stress model in a three-dimensional situation.

The process of comparing model predictions and experimental observation is applied between a twodimensional stress elasticity model of a ring compressed by two aligned forces [9] and the results of five different size tubes compressed by two flat surfaces. The measures are carried out by means of resistive strain gauges fixed on the external surface of the tube. The classical two-dimensional problem presented in this article has been already treated before $[10,11]$, but the uncertainty of the model and of the experiments results was not taken into account.

The benefit of using strain gauges is that it is possible to compare the resultant experimental slope $K_{E}$, known as the stress concentration factor and defined as $K_{E}=\sigma_{P} /(4 P / \pi L D)$, with the predictions proposed by Chianese [9]. The experimental stress concentration factor $K_{E}$, is the non-dimensional ratio between the tangential stress $\sigma_{P}$ and the stress $4 P / \pi l D$, where $P$ is the applied force; $D$ and $l$ are the external diameter and the tube length, respectively.

The experimental slope $K_{E}$ and its uncertainty are calculated considering the randomness of the variances in both Cartesian axes, $u^{2}\left(\sigma_{P}\right)$ and $u^{2}(4 P \pi L D)$, by means of a weighted least square algorithm [12]. The determination of the slope using this procedure enables us to reduce the comparison to a univariate analysis instead of comparing multiple stress states. 
Furthermore, an adaptive Monte Carlo procedure for the calculus of the theoretical model uncertainty is implemented [13], allowing thus to calculate the theoretical stress concentration factor and its uncertainty at any point of the ring. A global uncertainty model is proposed in order to improve the theoretical predictions, as it not only considers the stochastic behaviour of the model parameters but also the influence of the experimental parameters. This allows increasing the application range of the theoretical model when the ideal case is not met.

It is proposed in the paper to employ the $E_{N}$ number, a statistical magnitude used in proficiency testing for interlaboratory comparisons [14] as the criteria of model validation. For the model to be accepted as a good predictor of the real case under study, the $E_{N}$ number has to be between 0 and 1 . The $E_{N}$ number takes into account the difference between the results of the model and the experimental data as well as the magnitude of the global uncertainty model for a confidence level that has been demanded.

Moreover, the determination of a representative experimental sampling is usually conditioned by the high costs of achieving multiple trials, both in time and expenses [4]. Obviously, test repetitions and sample increments improve the reliability of the statistical analysis. That is why it is proposed that a resampling technique known as Bootstrapping, or sampling with replacements be used, as it is a simple and effective method to generate a bigger sampling when the initial sample size is small [15]. The advantage of this technique is that the probability distribution and the coverage interval is based on the experimental results and not assumed from the beginning [16]. The proposed methodology that combines the $E_{N}$ number with Bootsrapping will help decision-making when at least one sample does not meet the requirements of the $E_{N}$ number criteria. The possible solutions could be to repeat the test or to increase the sample size.

\section{Analytical Model}

The two-dimensional analytical solution of the circular ring with a concentric hole and rectangular cross section, which has been submitted to two symmetric and opposite forces (Figure 1), is obtained by the superposition of two stress

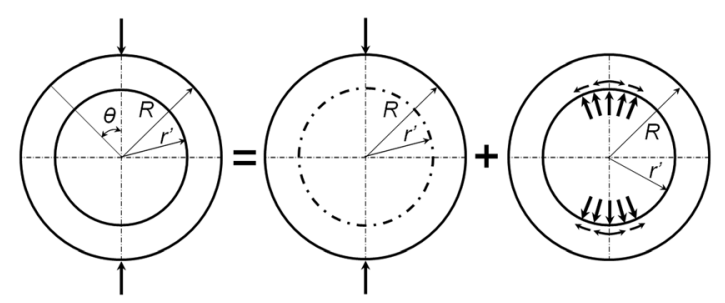

Figure I: Superposition of the stress in the compressed ring states [17]. The first state considers the stresses in a disc compressed by two equal and opposite forces, and the second accounts for a ring, where the stress in its inner rim cancels the stress in the disc of the same value of radius $r^{\prime}$ [18].

The procedure to find the exact solution of this twodimensional problem was first discussed by Timoshenko [19] and has been developed by Chianese and Erdlac [9] for any relationship between the radii and for any point of the ring. Other solutions were developed by authors like Nelson [20], Ripperger [10] and Batista [11], producing nearly identical results.

When the tangential stress distribution in a ring is studied, the stress concentration factor $K$ concept is normally used [19], as it is a dimensionless magnitude that depends exclusively on the radii ratio $\rho$ and the angle $\theta$ where the stress is calculated. Therefore, rings with different sizes but with the same $\rho$ present identical values of $K$.

According to Chianese and Erdlac [9], the tangential theoretical stress $\sigma_{P}$ for any point of the ring is expressed by

$\sigma_{P}=\frac{2 P}{\pi L R} K=\frac{4 P}{\pi L D} K$

where the stress concentration factor $K$ for the tangential stress is calculated solving the following equation [9]:

$$
\begin{aligned}
K= & \frac{1}{2}-\frac{1 \rho^{2}}{2 r^{2}}\left(\frac{1+r^{2}}{1-\rho^{2}}\right)-\frac{(1-r \cos \theta) \sin ^{2} \theta}{\left(r^{2}+1-2 r \cos \theta\right)^{2}}-\frac{(1+r \cos \theta) \sin ^{2} \theta}{\left(r^{2}+1+2 r \cos \theta\right)^{2}} \\
& +\sum_{n=2}^{\infty}\left[n(n-1) c_{n} r^{n-2}+(n+2)(n+1) d_{n} r^{n}+n(n+1) c_{n}{ }^{\prime} r^{-n-2}\right. \\
& \left.+(n-2)(n-1) d_{n}{ }^{\prime} r^{-n}\right] \cos n \theta
\end{aligned}
$$

and the coefficients of the infinite sum are defined as [9]

$$
\begin{aligned}
& c_{n}=\frac{1}{2(n-1) C}\left[n\left(\rho^{2}-1\right)+\left(\rho^{2 n}-1\right)-n^{2}\left(\rho-\frac{1}{p}\right)^{2}\right] \\
& d_{n}=\frac{1}{2(n+1) C}\left[n^{2}\left(\rho-\frac{1}{\rho}\right)^{2}+n\left(\frac{1}{\rho^{2}}-1\right)-\left(\rho^{2 n}-1\right)\right] \\
& c_{n}^{\prime}=\frac{1}{2(n+1) C}\left[n\left(\rho^{2}-1\right)+\left(\rho^{2 n}-1\right)\right] \\
& d_{n}^{\prime}=\frac{1}{2(n-1) C}\left[n\left(\rho-\frac{1}{\rho}\right)+\left(\rho^{2 n}-1\right)\right] \\
& C=n^{2}\left(\rho-\frac{1}{\rho}\right)^{2}-\left(\rho^{n}-\frac{1}{\rho^{n}}\right)^{2}
\end{aligned}
$$

This solution is expressed as a function of the ratio between the inner and outer radii $\rho$ equal to $r^{\prime} / R$ and the angular position $\theta$, which is the angle with respect to the point of load application. The index $n$ is always an even integer and the ratio $r$ defines the position of the point of interest in the cross section were the stress has to be 
calculated. This means that $r=\rho$ when the point is in the inner radius and $r=R / R=1$ when the point is in the outer radius.

Up to this date, the theoretical results developed by the authors cited before have only been compared with experimental results using hollow cylinders by means of photo-elasticity $[10,20]$. The authors discussed that the load distribution along the length of the tube is not really uniform, as it is approximately uniform except near the ends. Their results produced differences between the model and the experiment ranging from 0.2 to $3.64 \%$. Nevertheless, the uncertainties of the experiment and the model were not considered for the model validation.

The stress behaviour of real and finite tubes can then be approximated to the two-dimensional model but it is necessary to know how the ratio $L / D$ influences this approximation, and this has not yet been quantified.

\section{Methodology}

The experiment was initially designed with a sampling of five seamless alloy steel tubes E-355 of different diameters, thicknesses and lengths. In order to quantify the influence of the ratio $L / D$, the geometry of the tubes shown in Table 1 is such that they cannot be considered as long tubes. To ensure the uniformity of the diameters along their lengths and the concentricity, the turning of the outside diameter and the boring of the inside diameter was done in one set-up, that is, without moving the work between operations. The load was applied in such a way that, in none of the cases, the value of the yield strength of the tested material was exceeded.

The five tubes were instrumented with four tee rosettes of $6 \mathrm{~mm}$ gauge length with the disposition shown in Figure 2A. Rosette \#1 was located as close as possible to the tube border; rosette \#2 was in the middle of the tube and rosette \#3 was located between rosettes 1 and 2 . In order to control the symmetry in the application of the load, one additional strain gauge rosette (rosette \#4) was fixed in the middle of the length of the opposite side. For all the strain gauges, a three-wire quarter Wheatstone bridge circuit was employed [21].

The length of the strain gauges was selected knowing that its measurement is an average that occurred under its grip

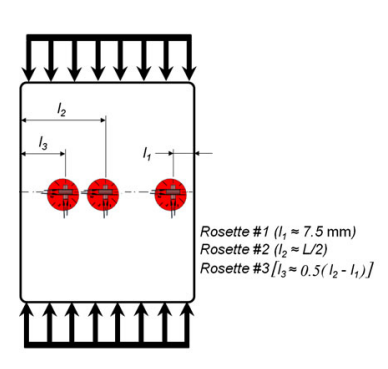

(A)

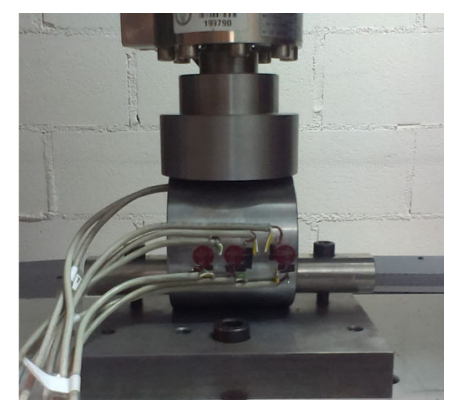

(B)
Figure 2: Distributions of the strain gauge rosettes in the compressed tube

[22]. Therefore, the theoretical stress concentration factor will be also integrated (Equation (3)), where $\Delta \alpha$ is the angle corresponding to the coverage of the gauge.

$\bar{K}=\frac{1}{\Delta \alpha} \int_{\Delta \alpha} K d \alpha$

The difference between the integrated value and the value for a single point is nevertheless superior to $0.03 \%$.

The tubes have been tested using a compression testing machine installed in a controlled climate room $\left(\sim 23^{\circ} \mathrm{C}\right)$. For the test, a compression plate and a base were especially manufactured and installed onto the force transducer and the machine test bench, respectively. The tube was fixed to the base in such a way that it was assured that it would not move (Figure 2B).

\section{Experimental data processing}

The force and the strain data were recorded at a $10 \mathrm{~Hz}$ frequency; the force applied $P$ was increased at a rate of $50 \mathrm{~N}$ until its maximum value was reached. Figure 3 shows the flowchart for the estimation of the experimental stress concentration factor $K_{E}$. This procedure was repeated 10 times for each tube.

All the sources of error inherent in the measurement of the strain gauges (Table 2) have been calculated and were subtracted from the final strain (Equation (4)) [22]. Then, the corrected strains were transformed into principal strains (Equation (5)) [23].

Table I: Tubes dimensions and applied compression loads

\begin{tabular}{|c|c|c|c|c|c|c|}
\hline Tube & Outer diameter $D(\mathrm{~mm})$ & Inner diameter $d(\mathrm{~mm})$ & Length L (mm) & $\rho=d / D$ & Compression Load P (kN) & Young Modulus E (Pa) \\
\hline I & 75.73 & 60.08 & 99.25 & 0.793 & 27.93 & $2.176 \times 10^{11}$ \\
\hline 2 & 98.47 & 82.32 & 87.68 & 0.836 & 20.11 & $2.063 \times 10^{11}$ \\
\hline 3 & 125.68 & 107.58 & 79.98 & 0.856 & 18.00 & $2.119 \times 10^{11}$ \\
\hline 4 & 149.60 & 132.82 & 80.08 & 0.888 & 12.96 & $2.130 \times 10^{11}$ \\
\hline 5 & 233.49 & 205.62 & 80.50 & 0.881 & 24.19 & $2.066 \times 10^{11}$ \\
\hline
\end{tabular}




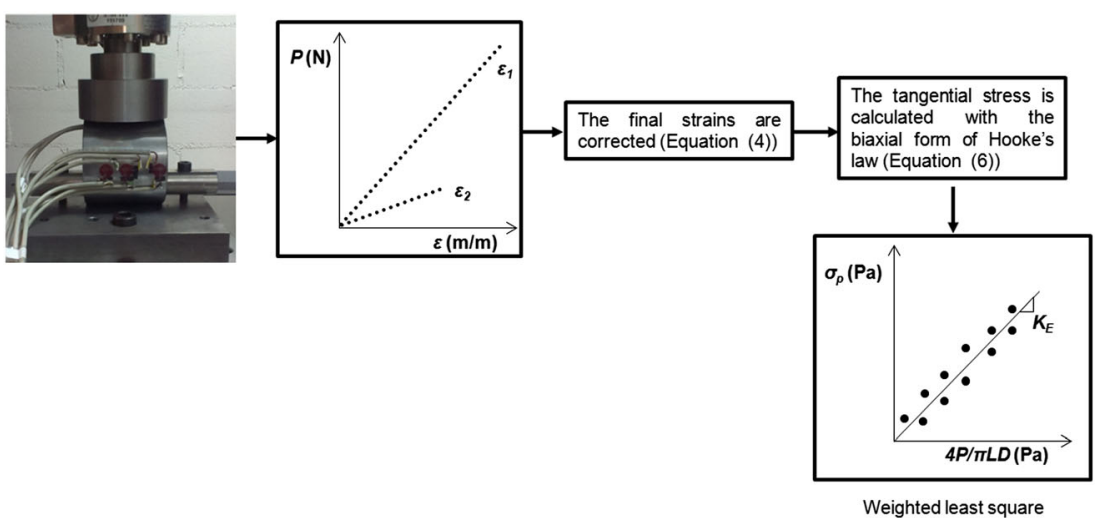

Figure 3: Flowchart for the estimation of the experimental stress concentration factor $K_{E}$

Table 2: Sources of error in strain gauges measurement

\begin{tabular}{|c|c|}
\hline Error due to & Formula \\
\hline $\begin{array}{l}\text { Wheatstone bridge } \\
\text { nonlinearity }\end{array}$ & $\begin{array}{l}E_{w, i}=\hat{\varepsilon}_{i}-\frac{2 \hat{\varepsilon}_{i}}{2-F \hat{\varepsilon}_{i}} \\
\varepsilon_{i:} \text { strain indicated by the strain data acquisition system } \\
\text { F: gauge factor. }\end{array}$ \\
\hline \multirow[t]{7}{*}{ Temperature } & $\begin{array}{l}E_{T, i}=\frac{2}{F\left[1+\alpha_{G}\left(T_{E}-T_{C}\right)\right]} \cdot\left[\varepsilon_{T / 0}+\left(\alpha_{S(B)}-\alpha_{S(A)}\right) \cdot\left(T_{E}-T_{C}\right)\right] \\
\text { 2: gauge factor used by the manufacturer to estimate the } \\
\text { thermal output of the strain gauge. }\end{array}$ \\
\hline & $\alpha_{G}$ : temperature coefficient of the gauge factor \\
\hline & $\varepsilon_{T / O}:$ thermal output or apparent strain \\
\hline & $\alpha_{s(B)}:$ coefficient of thermal expansion of the test material \\
\hline & $\begin{array}{l}\alpha_{s(A)} \text { : coefficient of thermal expansion of the material } \\
\text { used by the strain gauge manufacturer. }\end{array}$ \\
\hline & $\begin{array}{l}T_{E}, T_{C} \text { : Temperatures of the test piece and the room } \\
\text { temperature on the gauge package data label, respectively }\end{array}$ \\
\hline & $E_{T S, X}=\frac{K_{t}}{1-K_{t}^{2}} \cdot\left[\hat{\varepsilon}_{x}\left(v_{0}-K_{t}\right)+\hat{\varepsilon}_{y}\left(1-v_{0} K_{t}\right)\right]$ \\
\hline \multirow[t]{4}{*}{$\begin{array}{l}\text { Transverse } \\
\text { sensitivity }\end{array}$} & $E_{T S, Y}=\frac{K_{t}}{1-K_{t}^{2}} \cdot\left[\hat{\varepsilon}_{y}\left(v_{0}-K_{t}\right)+\hat{\varepsilon}_{X}\left(1-v_{0} K_{t}\right)\right]$ \\
\hline & $\begin{array}{l}\hat{\varepsilon}_{x}, \hat{\varepsilon}_{y} \text { : uncorrected strain of the gauges } \mid \text { and } 2 \text { of the } \\
\text { tee rosette }\end{array}$ \\
\hline & $K_{t}:$ transverse sensitivity coefficient \\
\hline & $v_{0}=0.285$ \\
\hline
\end{tabular}

$\varepsilon_{i}=\hat{\varepsilon}_{i}-E_{W}-E_{T}-E_{T S}$

$\varepsilon_{P, Q}=\frac{\varepsilon_{1}+\varepsilon_{2}}{2} \pm \frac{\varepsilon_{1}-\varepsilon_{2}}{2 \cos (2 \beta)}$

where $\varepsilon_{1}$ and $\varepsilon_{2}$ are the corrected strain gauge readings from the $x$ and $y$ axes, that are misaligned by an angle $\beta$ from the direction of the minimum and maximum strains $\varepsilon_{Q}$ and $\varepsilon_{P}$, respectively.

For the calculus of the experimental tangential stress $\sigma_{P}$, it is considered that each tube material is homogeneous and isotropic in its mechanical properties. Therefore, the biaxial form of Hooke's law is used to convert the principal strains from Equation (5) into the principal stresses (Equation (6)) [23].

$\sigma_{P}=\frac{E}{1-v^{2}}\left(\varepsilon_{P}+v \varepsilon_{Q}\right)$

where $E$ is the Young Modulus and $v$ is the Poisson ratio.

Afterwards, the tangential stress $\sigma_{P}$ was plotted versus the ratio $4 P / \pi L D$ and a linear relationship was observed. The final slope represents the experimental stress concentration factor, $K_{E}$.

\section{Determination of the experimental stress concentration factor and its uncertainty}

As the experimental stress concentration factor, $K_{E}$, is not measured directly, it is necessary to evaluate the uncertainty of different error sources to determine its uncertainty $U\left(K_{E}\right)$. The experimental stress concentration factor represents the slope between the tangential stress $\sigma_{P}$ and the ratio $4 P / \pi L D$ (Figure 3 ). Therefore, it is necessary to apply the law of propagation of uncertainties to calculate the variance of the axis $y\left(\sigma_{P}\right)$ and the axis $x(4 P / \pi L D)$, according to [24].

The Williamson-York method [12] is used to calculate the experimental stress concentration factor $K_{E}$. It is a weighted least square algorithm for calculating the best straight line when the variance in both axes exists [25]. The solution is obtained starting with an initial value of the slope that can be calculated by the standard least square method. Then, taking into account the influence of the variance of the axes in the initial slope, a new slope is calculated. The process is repeated until the difference between consecutive iterations is below the value of the desired accuracy. In this case, an accuracy of $10^{-6}$ was chosen, in order to ensure a high precision in the final slope. 
Uncertainty of the $y$-axis

The combined standard uncertainty of the corrected strains $\left(\varepsilon_{1}\right.$ and $\left.\varepsilon_{2}\right)$ was calculated with Equation (7), according to the expression developed by Montero et al. (Table 3) [26]:

$u\left(\varepsilon_{i}\right)=\sqrt{u^{2}\left(\hat{\varepsilon}_{i}\right)+u^{2}\left(E_{W, i}\right)+u^{2}\left(E_{T, i}\right)+u^{2}\left(E_{T S, i}\right)}$

Once the variance for each of the components forming Equation (7) is calculated, it is possible to obtain the combined variances of the principal strains (Equation (8)).

$u^{2}\left(\varepsilon_{P, Q}\right)=\left[\frac{\partial \varepsilon_{P, Q}}{\partial \varepsilon_{1}} u\left(\varepsilon_{1}\right)\right]^{2}+\left[\frac{\partial \varepsilon_{P, Q}}{\partial \varepsilon_{2}} u\left(\varepsilon_{2}\right)\right]^{2}+\left[\frac{\partial \varepsilon_{P, Q}}{\partial \beta} u(\beta)\right]^{2}$

As the magnitudes $E$ and $v$ have been obtained by simultaneous observations, a correlation between them exists [24]. Therefore, the combined variance of the tangential stress is calculated with the following equation:

$$
\begin{aligned}
u^{2}\left(\sigma_{P}\right) & =\left[\frac{\partial \sigma_{P}}{\partial \varepsilon_{P}} u\left(\varepsilon_{P}\right)\right]^{2}+\left[\frac{\partial \sigma_{P}}{\partial \varepsilon_{Q}} u\left(\varepsilon_{Q}\right)\right]^{2}+\left[\frac{\partial \sigma_{P}}{\partial E} u(E)\right]^{2} \\
& +\left[\frac{\partial \sigma_{P}}{\partial v} u(v)\right]^{2}+2 \frac{\partial \sigma_{P}}{\partial E} \frac{\partial \sigma_{P}}{\partial v} u(E, v)
\end{aligned}
$$

where

$u(E, v)=\frac{1}{n(n-1)} \sum_{i=1}^{n}\left(E_{i}-E\right)\left(v_{i}-v\right)$

\section{Uncertainty of the $x$-axis}

The uncertainty of the $x$-axis is determined by the force data acquisition system and the instrument used to geometrically characterise the tubes. As they were measured with the same calliper, the law of propagation of uncertainties and the correlation considered between $L$ and $D$ [27] produce the following expression of the $x$-axis combined variance:

$u^{2}(x)=\left[\frac{\partial x}{\partial P} u(P)\right]^{2}+\left[\left|\frac{\partial x}{\partial l} u(L)\right|+\left|\frac{\partial x}{\partial D} u(D)\right|\right]^{2}$
Uncertainty of the experimental stress concentration factor The experimental stress concentration factor $K_{E, i}$ and its uncertainty $u\left(K_{E, i}\right)$ for each $i$ repetition has been calculated using the weighted least squares algorithm [12]. Moreover, for each rosette, the final value of the experimental stress concentration factor is the mean of the $i$ th observations $(n=10)$ made, i.e.:

$\bar{K}_{E, r}=\frac{1}{n} \sum_{i=1}^{n} K_{E, i}$

So the standard uncertainty associated with $\bar{K}_{E, r}$ (Equation (14)) considers the contribution of the two uncertainty of each observation (Equation (12)) and the experimental standard deviation of $\bar{K}_{E, r}$ (Equation (13)).

$u\left(\bar{K}_{E}\right)=\frac{1}{n} \sum_{i=1}^{n} u\left(K_{E, i}\right)$

$s^{2}\left(\bar{K}_{E, r}\right)=\frac{\sum_{i=1}^{n}\left(K_{E, i}-\bar{K}_{E, r}\right)^{2}}{n(n-1)}$

$u\left(\bar{K}_{E, r}\right)=\sqrt{u\left(\bar{K}_{E}\right)+s^{2}\left(\bar{K}_{E, r}\right)}$

For a 95\% confidence level, the expanded uncertainty is [24]:

$U\left(\bar{K}_{E, r}\right)=2 u\left(\bar{K}_{E, r}\right)$

It has to be reminded that the extrapolation of the twodimensional model for hollow cylinders is based on the simplification of considering a uniform stress distribution along the tube length. In order to quantify the contribution of this approximation, the experimental stress concentration factor is averaged for each tube $\left(\bar{K}_{E}\right)$, and the uncertainty $u\left(\bar{K}_{E, a v}\right)$ due to this averaging has been computed, considering that all the possible values of the experimental stress

Table 3: Variance of the strain measurement

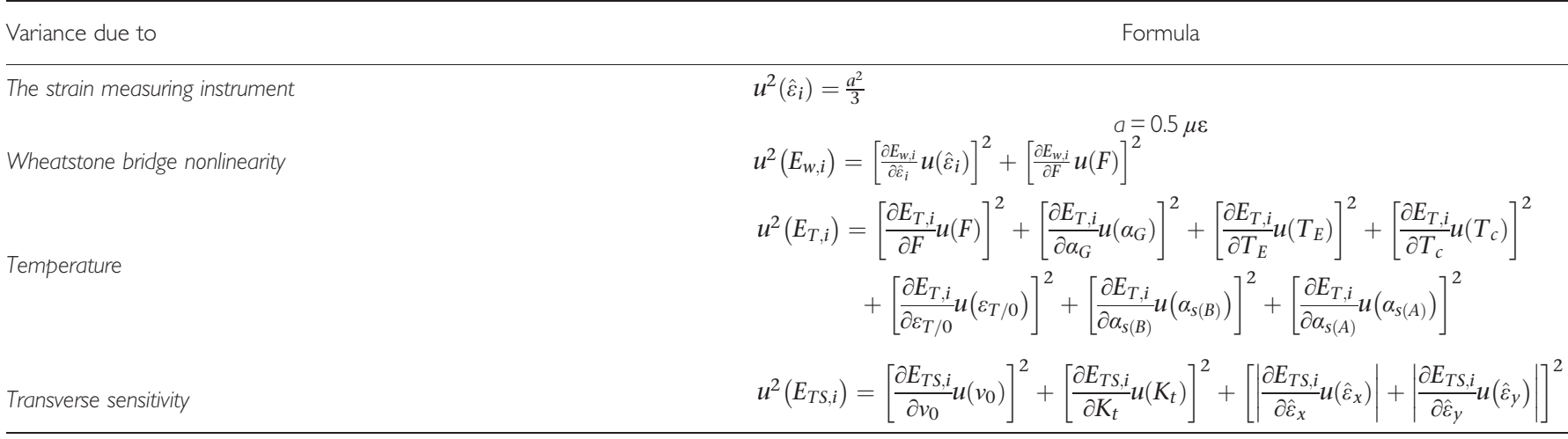


concentration factor are between the maximum and minimum calculated for each tube [13]. $u\left(\bar{K}_{E, a v}\right)$ is thus expressed by the following rectangular distribution:

$u\left(\bar{K}_{E, a v}\right)=\frac{\bar{K}_{E, r \max }-\bar{K}_{E, r \min }}{\sqrt{12}}$

$u\left(\bar{K}_{E, a v}\right)$ can thus be an indicator of how far the analysed specimen is from the ideal condition of the model. The error associated to this concept cannot be determined and it is the reason why it is considered as zero and computed as an uncertainty.

In order to minimise the magnitude of uncertainties when it was possible, the Young's modulus $E$ and the Poisson ratios $v$ of each tube have been determined using flat specimens taken from the tubes [28]. The isotropy and homogeneity is therefore considered within each tube but not within the sample for the sake of better accuracy. The tests were repeated five times on each sample and performed as recommended by ASTM [29, 30]. Their uncertainties were calculated following the recommendation of Gabauer [31] computing the variance of the mean and the uncertainties of the measurement instruments.

Table 4 summarises all the variables used to calculate the experimental stress concentration factor and its uncertainty. Several uncertainties were calculated by means of type B evaluation, assuming a symmetric rectangular distribution [24]. Note that some of the variables have been presented in the following form: for example, the Young Modulus is

Table 4: Values and uncertainty of all the variables

\begin{tabular}{|c|c|c|c|c|c|}
\hline Variable & Value & Units & Tolerance & Semi-range $a$ & Uncertainty $u$ \\
\hline$F$ & 2.10 & Dimensionless & $\pm 1.00 \times 10^{-2}$ & $1.00 \times 10^{-2}$ & $5.77 \times 10^{-3}$ \\
\hline$K_{t}$ & $1.00 \times 10^{-3}$ & Dimensionless & $-1.00 \times 10^{-3}$ & $1.00 \times 10^{-3}$ & $5.77 \times 10^{-4}$ \\
\hline$\alpha_{G}$ & $1.00 \times 10^{-2}$ & ${ }^{\circ} \mathrm{C}^{-1}$ & $\pm 5.00 \times 10^{-5}$ & $5.00 \times 10^{-5}$ & $2.89 \times 10^{-5}$ \\
\hline$v_{0}$ & 0.285 & Dimensionless & $\pm 1.00 \times 10^{-2}$ & $1.00 \times 10^{-2}$ & $5.77 \times 10^{-3}$ \\
\hline$\beta$ & $-1.05 \times 10^{-2} \leq \beta \leq 1.75 \times 10^{-2}$ & Radians & $\pm 8.73 \times 10^{-4}$ & - & $9.60 \times 10^{-4}$ \\
\hline L & See Table I & $\mathrm{mm}$ & $\pm 1.00 \times 10^{-1}$ & - & $2.15 \times 10^{-2}$ \\
\hline \multicolumn{6}{|l|}{$D$} \\
\hline d & See Table I & $\mathrm{mm}$ & $\pm 5.00 \times 10^{-1}$ & - & $2.89 \times 10^{-2}$ \\
\hline$T_{E, I}$ & 21 & ${ }^{\circ} \mathrm{C}$ & \pm 1.00 & 1.00 & $5.77 \times 10^{-1}$ \\
\hline$T_{E, 2}$ & 21 & & & & \\
\hline$T_{E, 3}$ & 23 & & & & \\
\hline$T_{E, 4}$ & 25 & & & & \\
\hline$T_{E, 5}$ & 21 & & & & \\
\hline$\varepsilon_{T / O, I}$ & $3.80 \times 10^{-1}$ & $\mu \mathrm{m} / \mathrm{m}$ & - & - & $8.50 \times 10^{-1}(\mu \mathrm{m} / \mathrm{m}) /{ }^{\circ} \mathrm{C}$ \\
\hline$\varepsilon_{T / O, 2}$ & $3.80 \times 10^{-1}$ & & & & \\
\hline$\varepsilon_{T / 0,3}$ & $8.96 \times 10^{-1}$ & & & & \\
\hline$\varepsilon_{T / O, 4}$ & 1.09 & & & & \\
\hline$\varepsilon_{T / 0,5}$ & $3.80 \times 10^{-1}$ & & & & \\
\hline P & Varies & $\mathrm{kN}$ & - & - & $2.5 \times 10^{-3} \mathrm{P}$ \\
\hline$T_{C}$ & 23 & ${ }^{\circ} \mathrm{C}$ & - & - & - \\
\hline$\alpha_{S(A)}$ & $11.8 \times 10^{-6}$ & ${ }^{\circ} \mathrm{C}^{-1}$ & - & - & - \\
\hline$\alpha_{s(B)}$ & $11.7 \times 10^{-6}$ & ${ }^{\circ} \mathrm{C}^{-1}$ & - & - & - \\
\hline \multicolumn{6}{|c|}{ Values and uncertainty of the test material using type $A$ evaluation } \\
\hline$E_{1}$ & $2.176 \times 10^{11}$ & $\mathrm{~Pa}$ & - & - & $1.95 \times 10^{9}$ \\
\hline$E_{2}$ & $2.063 \times 10^{11}$ & & - & - & $6.14 \times 10^{8}$ \\
\hline$E_{3}$ & $2.119 \times 10^{11}$ & & - & - & $1.79 \times 10^{8}$ \\
\hline$E_{4}$ & $2.130 \times 10^{11}$ & & - & - & $4.77 \times 10^{8}$ \\
\hline$E_{5}$ & $2.066 \times 10^{11}$ & & - & - & $1.79 \times 10^{8}$ \\
\hline$v_{1}$ & $3.010 \times 10^{-1}$ & Dimensionless & - & - & $2.03 \times 10^{-4}$ \\
\hline$v_{2}$ & $3.000 \times 10^{-1}$ & & - & - & $2.00 \times 10^{-4}$ \\
\hline$v_{3}$ & $3.043 \times 10^{-1}$ & & - & - & $2.05 \times 10^{-4}$ \\
\hline$v_{4}$ & $2.909 \times 10^{-1}$ & & - & - & $2.98 \times 10^{-4}$ \\
\hline$v_{5}$ & $2.893 \times 10^{-1}$ & & - & - & $1.11 \times 10^{-4}$ \\
\hline
\end{tabular}


$E_{i}$ where $i$ represents the number of the tube where the variable was measured.

\section{Procedure to determine the uncertainty of the theoretical stress concentration factor}

An adaptive Monte Carlo procedure [13] was implemented for the uncertainty calculus of the theoretical stress concentration factor. The adaptive procedure for the case study is shown in Figure 4 and it allows solving a calculus in a few minutes that could take several hours due to the infinitive summation needed.

The adaptive method is based on carrying out an increasing number of Monte Carlo trials $(h)$ until the results of interest have stabilised. The result is deemed to have stabilised if twice the standard deviation associated with the stress concentration factor, its uncertainty and its left and right hand of the coverage intervals $\left(2 s_{K}, 2 s_{u(K)}, 2 s_{K_{L}}, 2 s_{K_{H}}\right)$ are less than the numerical tolerance associated with the standard uncertainty $u(K)$, in this case $\delta=0.510^{l}$, according to the Guide to the expression of Uncertainty in Measurement (GUM) [13], where $l$ represents the position of the $n_{d i g}$ digit integers associated with the standard uncertainty $u(K)$.

When the numerical tolerance $\delta$ is reached, all $h \times M$ model values obtained are used to calculate the theoretical stress concentration factor $\bar{K}$ and its uncertainty $u\left(\bar{K}_{M C}\right)$. The inputs of the theoretical stress concentration factor are
$D, d$ and $\theta$. As their uncertainties are known (Table 4), a Gaussian probability distribution function was selected. Therefore, according to the Guide to the expression of uncertainty in measurement [13], a coverage factor of two is used, corresponding to a coverage probability of approximately $95 \%$.

\section{Theoretical model enhancement by means of uncertainty}

The uncertainty analysis and quantification offers a possibility to enhance the two-dimensional model taking into account the real three-dimensional effects. The variations of the experimental concentration factor along the tube length, depicted by $u\left(\bar{K}_{E, a v}\right)$, are due to real effects that are not considered in the two-dimensional and ideal theoretical model. So, if the ideal conditions are not met, this paper proposes an increase in the uncertainty calculated for the elasticity model $u\left(\bar{K}_{M C}\right)$ with the addition of the $u\left(\bar{K}_{E, a v}\right)$. Therefore, the standard uncertainty associated to the model will be

$u(\bar{K})=\sqrt{u^{2}\left(\bar{K}_{M C}\right)+u^{2}\left(\bar{K}_{E, a v}\right)}$

With this approach, the results predicted by the model are not changed but are improved when the assumption of uniform stress along the length diverges from the real situation.

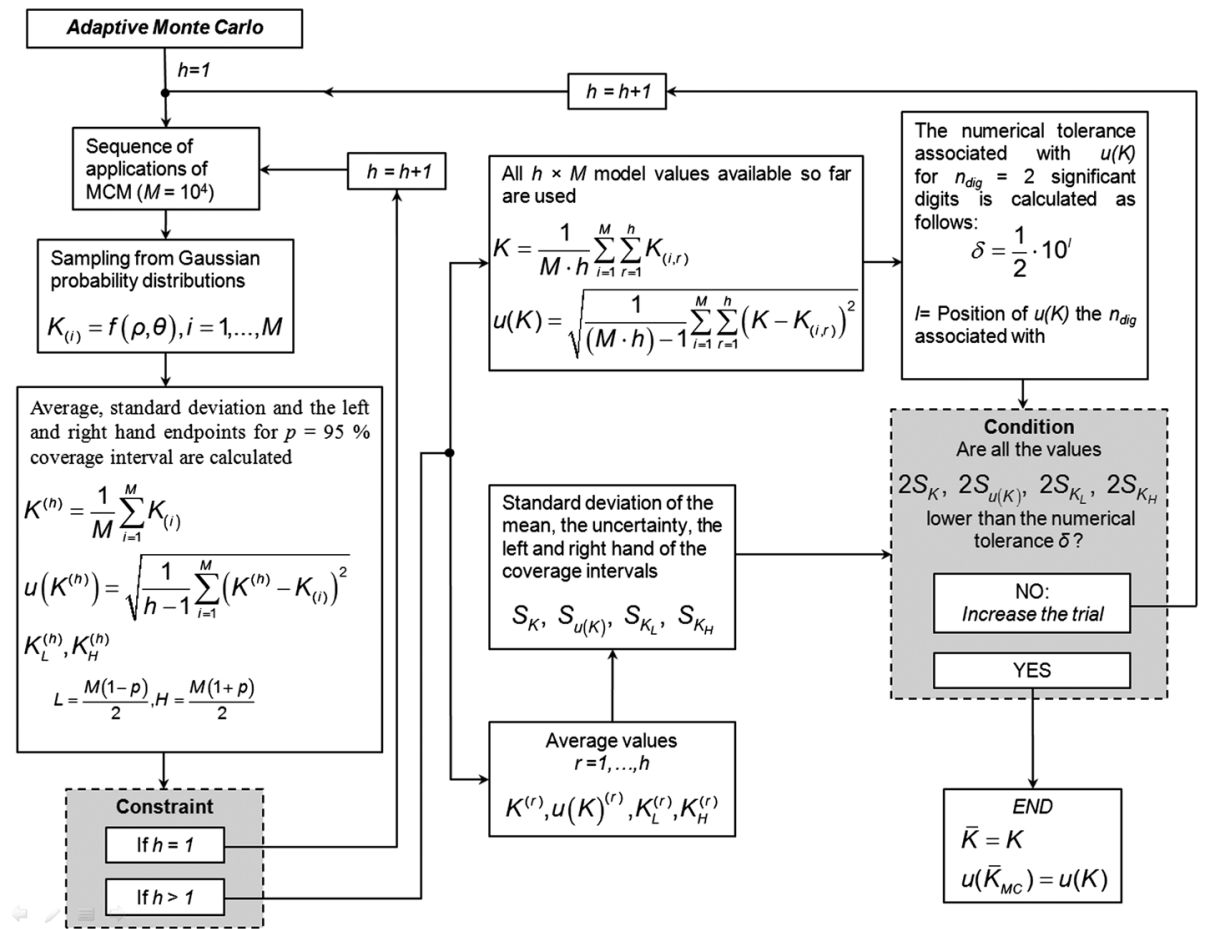

Figure 4: Adaptive Monte Carlo procedure for the evaluation of the uncertainty of the theoretical stress concentration factor $K$, according to Guide to the expression of uncertainty in measurement [13] 


\section{Bias and uncertainty between model and experiment}

The level of compliance of a model with a real given case is only fully quantified if both experimental and theoretical results are presented with their correspondent uncertainty. Nevertheless, the criteria or tolerance for its acceptance has to be decided by the engineers according to the accuracy required in their work field, dealing with the conflict between structural integrity and cost optimizations [3].

Model validation is normally performed using the null hypothesis [7], where, for a given level of confidence, the results of the prediction and the experiment are compared. Assuming a normal distribution, it is said that the predictions are within the range of probable values, that is, they are good estimators of reality, if the critical statistic calculated with the $t$ or $z$-test, is between the proposed range. In the case of the stress concentration factor, the critical parameter to be calculated with the $z$-test is

$z_{\text {critical }}=\frac{\left|\bar{K}-\bar{K}_{E}\right|}{\sqrt{u\left(\bar{K}_{E}\right)^{2}+u(\bar{K})^{2}}}=\frac{\mid \text { Error } \mid}{u(\text { Error })}$

So that if the $z_{\text {critical }}$ value of Equation (18) is between the $z$ $\pm z_{\alpha / 2}$ of the $z$ distribution for a probability $\alpha$, corresponding to a confidence interval of $100(1-\alpha) \%$, it is concluded that there is not enough statistical evidence to reject the model.

As confidence levels of $95 \%$ are usually defined in the fields of statistical validation and metrology [4, 24], it is proposed to calculate the $E_{N}$ number, normally used in interlaboratory comparisons [14], in order to accept or refuse the predictions (Equation (19)). An $E_{N}$ number equal to one, for a confidence level of $100(1-\alpha) \%$ corresponds to the statistics $z \pm Z_{\alpha / 2}$ in the case of the $z$ distribution. This is because $E_{N}$ numbers are calculated using expanded uncertainties in the denominator instead of standard deviations as $z_{\text {critical }}$

$E_{N}=\frac{\mid \text { Error } \mid}{U(\text { Error })}=\frac{\mid \text { Error } \mid}{k \cdot u(\text { Error })}=\frac{Z_{\text {critical }}}{k}$

where $k$ is the coverage factor corresponding to the confidence level required.

So if the uncertainties are estimated in a way consistent with the Guide to the expression of uncertainty in measurement, $E_{N}$ expresses the validity of the expanded uncertainty associated with each result [14].

A value of $E_{N}$ number smaller than the unit will mean that the expanded uncertainty of the difference is bigger than the difference itself, so the uncertainty fields have a part in common and it can be inferred that both results are compatible, and thus represent the same measurand. On the other hand, if it is higher than the unit, it is because the difference is bigger than its uncertainty having no common parts and thus it is said that they are not reproducing the same measurand, so the model needs to be corrected or improved.

\section{The bootstrap method as indicator of the consistency of the results}

Even under controlled circumstances, experimental procedures can be submitted to errors and it can happen that within the sampling chosen, some of the experimental results match with the model but others don't. Moreover, in engineering research, the samplings are usually small as they can be expensive, time consuming and even destructive.

The paper proposes the use of a statistical resampling technique known as bootstrap method [16] to calculate the coverage interval of the sampling results, when, as in this case, the sample size is insufficient for straightforward statistical inference [32]. The use of this method, together with the $E_{N}$ number, allows obtaining a frequency distribution and a coverage interval representing the sampling. The decision on whether the sampling enables the model validation can therefore rely on the values of the coverage interval limits depending on whether they are inferior or superior to one.

In this case, bootstrapping is applied to a sample of $5 E_{N}$ numbers as follows:

- Generate $B$ bootstrap samples of five elements each by means of random replacement. The number of $B$ samples being normally between 1000 and 2000 [33], and if a higher precision is required, it is recommended to use a bigger size of $B=10000$ samples [34] as in this case.

- Calculate the statistic of interest for each $B$ bootstrap sample. In this case, the average is chosen.With the 10000 values in increasing order [33], represent the frequency distribution and calculate the inferior and superior limit, for a coverage interval of $100 p \%$, where:

$E_{N L}=\bar{E}_{N(r)}$
$E_{N H}=\bar{E}_{N(r+q)}$

With $r=(n-q) / 2, q=p n$ and $p=0.95$

The value of $r$ has to be an integer; if not, a conservative value would be rounded off as $r=(n-q+1) / 2$ [13].

\section{Results and Discussion}

Following the steps indicated in the methodology, it is necessary to quantify the values of the error sources described in Table 2, in order to calculate the experimental stress concentration factor $\bar{K}_{E}$.

The maximum error in strain measurements due to several sources was inferior to $1 \mu \varepsilon$ representing around $0.1 \%$ of the maximum strains recorded $(\approx 850 \mu \varepsilon)$ on the tube borders. Even though the error values have remained 
relatively low, they were not discarded, i.e. all the strains recorded have been corrected in order to obtain the highest possible accuracy in the calculation of the experimental stress concentrator factor $\bar{K}_{E}$.

No significant differences between the experimental results from the rosettes placed on opposite sides (rosettes \#2 and \#4) were observed (Table 5). It is thus confirmed that the load was aligned with the tubes during the tests.

On the other hand, it was observed that the distribution of the stress concentration factor along the tube length presented less variation when the ratio length vs diameter $(L / D)$ increased. Figure 5 shows the longitudinal distribution of the experimental stress concentration factor for the tubes with the lower and higher variation, tubes 1 and 5 , respectively. In order to compare the results, the gauge positions is given in relative terms.

Tube 5 presents a higher discrepancy for longitudinal results; it was observed that the gauge closer to the edge increases its value up to $7.8 \%$ with respect to the gauge in the middle of the tube length. This can be caused by the fact that as the $L / D$ ratio decreases, the tube resembles less to the ideal case so the border effect produces a higher and negative influence on the uniformity of the stress distribution.

Table 5: Final experimental stress concentration factor $K_{E}$ for rosette \#2 and \#4

\begin{tabular}{lrrrrr}
\hline & Tube I & Tube 2 & Tube 3 & Tube 4 & Tube 5 \\
\hline Rosette \#2 & 30.38 & 51.53 & 64.66 & $1 \mid 4.02$ & 101.21 \\
Rosette \#4 & 30.35 & 51.52 & 64.40 & 113.99 & 101.19 \\
$\%$ Diff $=100 \frac{\text { max-min }}{\max }$ & 0.10 & 0.01 & 0.40 & 0.03 & 0.02 \\
\hline
\end{tabular}

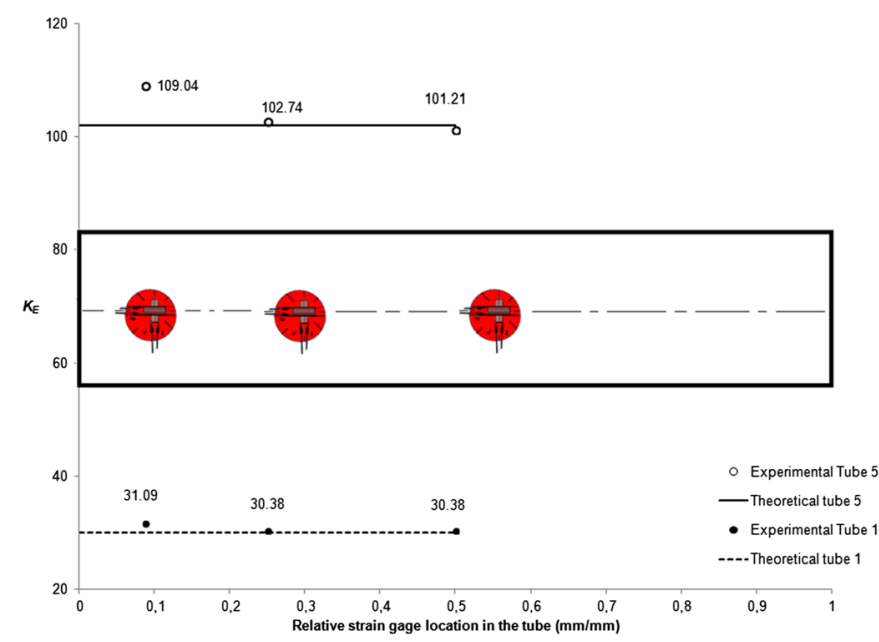

Figure 5: Distribution of the experimental stress concentration factor with the tube length
Table 6 shows the value of uncertainties associated with the experimental stress concentration factor, for each of the tubes analysed. It can be seen that the uncertainty due to the weighted least square results greater than the one calculated by the repeatability in the majority of the cases.

Moreover, the uncertainty due to the variation along the length $u\left(\bar{K}_{E, a v}\right)$ presents the highest magnitude of all and can be explained by the negative influence of the border effect as shown in Figure 6.

As can be observed in Figure 6, the evolution of the uncertainty due to the variation of the stress along the length presents an exponential function behaviour with respect to $L / D$. It also demonstrates that for ratios $L / D>1.2$, the magnitude of $u\left(\bar{K}_{E, a v}\right)$ reaches values of the same order than the other components of experimental uncertainty (Table 6). When the tube is of infinite length, its contribution will be nearly zero; this evolution being consistent with the principles of the elasticity model.

Table 7 shows the mean experimental stress concentration factor $\bar{K}_{E}$ for the five tubes and the respective theoretical values of $\bar{K}$ calculated with Equation (3).

Table 6: Uncertainty of the experimental stress concentration factor $\bar{K}_{E}$

\begin{tabular}{ccccc}
\hline Tube & $\begin{array}{c}\text { Weighted } \\
\text { least square } \\
\text { Equation } \\
(12)\end{array}$ & $\begin{array}{c}\text { Experimental standard } \\
\text { deviation of the mean }\end{array}$ & $\begin{array}{c}\text { Uncertainty } \\
\text { of } \bar{K}_{E}\end{array}$ & $\begin{array}{c}\text { Average } \\
\text { along the } \\
\text { length }\end{array}$ \\
\hline 1 & 0.15 & 0.17 & $\begin{array}{c}\text { Equation } \\
(14)\end{array}$ & $\begin{array}{c}\text { Equation } \\
(16)\end{array}$ \\
2 & 0.12 & 0.10 & 0.22 & 0.22 \\
3 & 0.17 & 0.04 & 0.16 & 0.45 \\
4 & 0.18 & 0.12 & 0.17 & 0.80 \\
5 & 0.06 & 0.31 & 0.22 & 1.02 \\
\hline
\end{tabular}

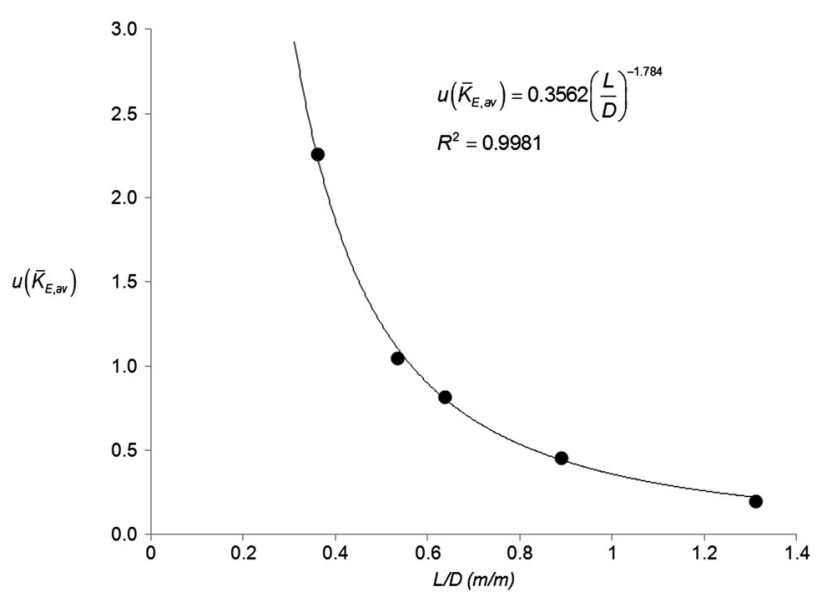

Figure 6: Evolution of $u\left(\bar{K}_{E, a v}\right)$ with the ratio $L / D$ 
Table 7: Final results of the experimental stress concentration factor, theoretical values, error and experimental uncertainty for a coverage factor of $k=2$

\begin{tabular}{|c|c|c|c|c|c|}
\hline & $\begin{array}{c}\text { Tube I }(\rho=0.793) \\
(L / D=|.3|)\end{array}$ & $\begin{array}{c}\text { Tube } 2(\rho=0.836) \\
\quad(L / D=0.89)\end{array}$ & $\begin{array}{c}\text { Tube } 3(\rho=0.856) \\
(L / D=0.64)\end{array}$ & $\begin{array}{l}\text { Tube } 4(\rho=0.888) \\
\quad(L / D=0.54)\end{array}$ & $\begin{array}{c}\text { Tube } 5(\rho=0.88 I) \\
(L / D=0.36)\end{array}$ \\
\hline$\overline{\bar{K}_{E}}$ & 30.61 & 51.90 & 66.18 & $1|6.0|$ & 104.33 \\
\hline$U\left(\bar{K}_{E}\right)$ & 0.44 & 0.31 & 0.35 & 0.43 & 0.63 \\
\hline $\bar{K}$ & 29.97 & 50.75 & 67.79 & 116.98 & 102.25 \\
\hline$U(\bar{K})$ & 0.53 & 1.05 & 1.69 & 2.25 & 4.56 \\
\hline Error $=\bar{K}-\bar{K}_{E}$ & -0.64 & -1.15 & 1.61 & 0.97 & -2.08 \\
\hline$\%$ Error $=100$ Error $\bar{K}$ & -2.13 & -2.26 & 2.37 & 0.83 & -2.03 \\
\hline$E_{N}=\frac{\mid \text { Error } \mid}{U(\text { Error })}$ & 0.92 & 1.05 & 0.93 & 0.42 & 0.45 \\
\hline
\end{tabular}

The relative difference between the experimental and the theoretical result varies from $0.83 \%$ for tube 4 to $2.37 \%$ for tube 3. From a quantitative point of view, it can be inferred that the differences are small and coincident with the ranges found by other authors [20]. Moreover, the differences are not showing any particular tendency with respect to the geometry of the tubes, so it can be assumed that the differences are due to uncontrolled phenomena and/or the difference between an ideal two-dimensional model and a real specimen.

It can also be seen in Table 7 how the uncertainty of the theoretical model $U(\bar{K})$ increases when the $L / D$ ratio decreases, due to the negative effect of the border in the assumption of uniform stress along the tube length.

As for the influence of the variables considered in the theoretical model, it has been observed that the uncertainty $u\left(\bar{K}_{M C}\right)$ increases as the radii ratio $\rho$ also increases: the minimum value is found for tube $1 ; u\left(\bar{K}_{M C}\right)=0.15$ and the maximum for tube 4 with $u\left(\bar{K}_{M C}\right)=0.47$. The stress model would be, hence, presenting a higher uncertainty $U(\bar{K})$ for a thin wall tube with a big diameter compared to its length.

Following the indication of Sandia [4], a global uncertainty, composed by the theoretical and experimental uncertainties, is represented around the theoretical result and is calculated with the following equation:

$$
U\left(K_{\text {global }}\right)=2 \sqrt{u^{2}(\bar{K})+u^{2}\left(\bar{K}_{E}\right)}
$$

This allows to clearly depict if the experimental result is within the range of possible values given by the global uncertainty, and avoids false positives when the ranges $U(\bar{K})$ and $U\left(\bar{K}_{E}\right)$ have a certain level of coincidence without this meaning that the model prediction agrees with the experimental result.

Figure 7 shows the graphical representation of the $E_{N}$ number and the $U\left(K_{\text {global }}\right)$, providing an objective and visual criteria. If the experimental value falls within the range
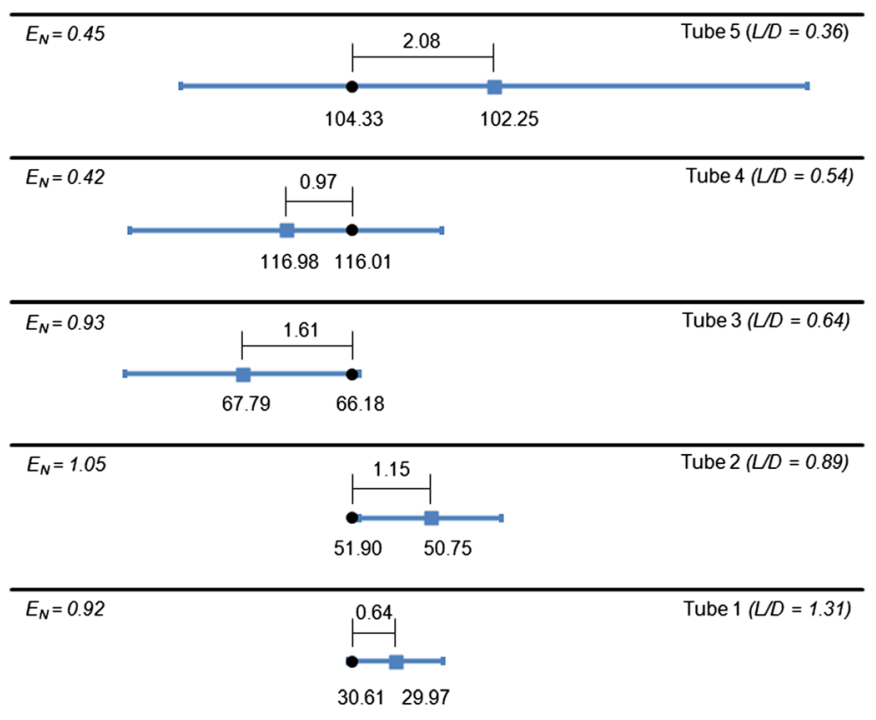

- Experimental results

Theoretical results

Figure 7: Differences between experimental and theoretical results for each of the tubes tested with the global expanded uncertainty of the model, $U\left(K_{\text {global }}\right)$

given by the global uncertainty, the model is considered valid for the given confidence level.

It can also be seen (Figure 7) that four of the five tubes present $E_{N}$ numbers inferior to one for a confidence level of $95 \%$. The $E_{N}$ number of tube 2 is slightly superior to one, whereas the numbers of tubes 1 and 3 are inferior but very close to it. When the $L / D$ ratio decreases, the exponential increase of $u\left(\bar{K}_{E, a v}\right)$ uncertainty generates an $E_{N}$ number that is clearly inferior, as happens with tubes 4 and 5 .

This could be initially considered to be a contradiction, as it may appear that the shorter tubes tested behave better than the longer ones when the latter are closer to the ideal condition of long tubes.

However, this first analysis needs a deeper reasoning as when the tube tested is longer, the uncertainty $u\left(\bar{K}_{E, a v}\right)$ decreases exponentially and so does the global uncertainty 
$U\left(K_{\text {global }}\right)$. As a consequence, any small variation in the error makes the $E_{N}$ number get quickly close to one or be even superior to it. As a general rule, it can be said that if the global uncertainty is small because the case under study is close to the ideal model, the model parameters are well characterised and the measurement device is accurate, the tests have to be performed under very controlled conditions to minimise the error and thus reach an acceptable $E_{N}$ value.

Moreover, it has to be pointed out that an $E_{N}$ number superior to one doesn't necessarily mean that the model has to be rejected. The $E_{N}$ number indicates how far the theoretical result from the experimental one is. After that, the final decision to accept or reject the model will depend on the engineer and on the margin of error he can consider in his design, what is generally called an engineering validation [4].

The case under study in this paper presents a situation where the sampling is relatively small and one of the tested tubes did not fulfil the criteria. The application of the bootstrap method according to point 2.6 provides an average $E_{N}$ number $\left(\bar{E}_{N}\right)$ and a coverage interval of the sampling $\left(\bar{E}_{N L}, \bar{E}_{N H}\right)$. Figure 8 presents the probability distribution obtained for 10000 bootstrap samples.

As can be seen, both the average $E_{N}$ number $\left(\bar{E}_{N}\right)$ and the coverage interval $\left(\bar{E}_{N L}, \bar{E}_{N H}\right)$ are inferior to one, so it can be concluded that the model prediction is consistent with the case under study even if one of the five tubes was out of the criteria.

In order to demonstrate how the proposed procedure can be a useful decision-making tool, and also as the superior limit $\bar{E}_{N H}$ is quite close to one, a new tube was prepared out of the same specimen from which tube 2 was mechanised. The sampling has therefore been increased from five to six tubes.

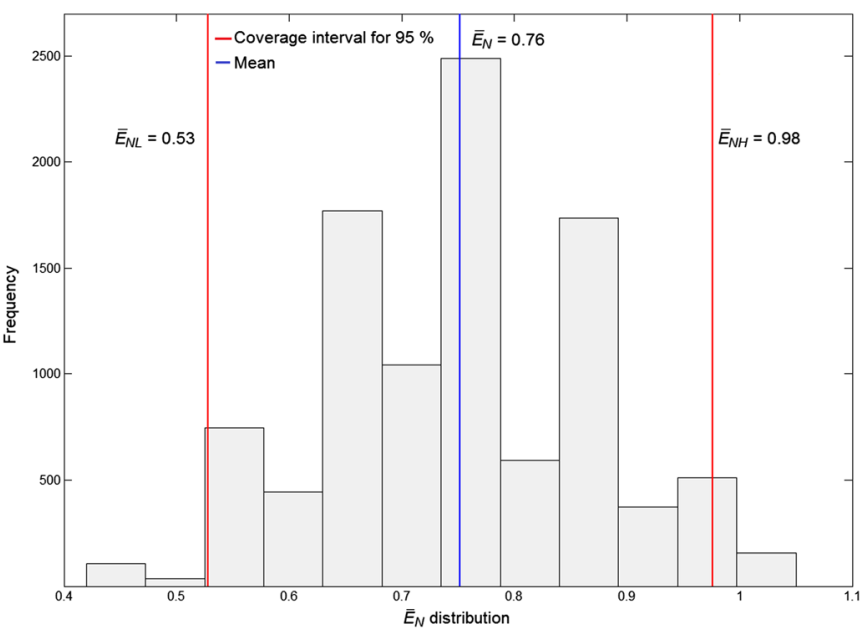

Figure 8: Bootstrapping distribution of the ${ }_{N}{ }^{-}$samples
The dimensions of tube 6 are $D=99.93 \mathrm{~mm}, d=85.76 \mathrm{~mm}$ and $L=100.55 \mathrm{~mm}$. A load of $12 \mathrm{kN}$ was applied obtaining experimental and theoretical concentration factors of $\bar{K}_{E}=$ $78.44 \pm 0.53$ and $\bar{K}=77.73 \pm 0.99$, respectively, and an $E_{N}=0.63$. This value will have a positive effect in the general sampling behaviour. The bootstrapping was repeated again also considering tube 6 in the sample and obtaining an average $E_{N}$ number $\bar{E}_{N}=0.73$ and a coverage interval of $(0.54,0.92)$.

\section{Conclusions}

This paper presents a new procedure based on a three-step validation procedure (TSVP) that allows to quantify the application limits of a two-dimensional stress model of a ring compressed by two aligned forces [9] for the case of five different size tubes, compressed by two flat surfaces. The measurements are carried out by means of resistive strain gauges fixed on the external surface of the tube.

The first step of the validation procedure is the development of a global uncertainty budget. This requires a good knowledge of the parameters that influence more both the model and the experiment and also of the latest statistical procedures considered in metrology [13, 24] as the ones used in this paper.

Moreover, in this case under study, the stress behaviour of real and finite tubes have been approximated to the twodimensional model considering the uncertainty of the stress variation along the length because of the border effect and whose expression is an exponential function of the $L / D$ ratio.

Many authors have promoted the use of statistical tools and uncertainties to validate theoretical models [3-5,35]. This paper proposes the calculus of the $E_{N}$ number as the second step of the procedure. The $E_{N}$ number is a statistical magnitude used in interlaboratory comparisons, with a confidence level of $95 \%$, this meaning that values of $E_{N}<1$ indicate that model predictions are compatible with the experimental results [14]. Four of the five tubes fulfilled the $E_{N}$ criteria and the longer ones produced $E_{N}$ values closer to one. This can happen when the uncertainties are small due to a good match between the model and the experiment and a good control of the parameter magnitudes. In this situation, a small variation in the error makes the $E_{N}$ number get quickly closer to one or be even superior to it.

In order to determine the weight of the failed test in the global sampling behaviour, it is proposed to use the bootstrap method to determine the interval of confidence of the bootstrapped sample as the third and last step of the methodology. Bootstrapping will determine if more tests are needed thus providing a useful decision-making tool. In this case, despite the bad result of tube 2, the global coverage limits are below the unit. Still, it was decided to 
increase the sample analysed, by testing a new tube that provided a new $E_{N}$, which improved the coverage limits, thereby showing the advantage of bootstrapping in decision-making, when the sample is small.

The three-step procedure (TSVP) has offered good results for the case that has been studied in the paper as the use of the two-dimensional model is validated for the tubes tested, and the validation could thus be extrapolated to other tubes with similar $\rho$ and $L / D$ ratios. Further research can be undertaken to apply the TSVP in other structural cases.

\section{References}

1. Elishakoff, I. (2013) Recent developments in applied mechanics with uncertainties. 4th Inverse Problems, Design and Optimization Symposium (IPDO-2013).

2. Elishakoff, I., and Ohsaki, M. (2010) Optimization and AntiOptimization of Structures Under Uncertainty. World Scientific Publishing Company, Incorporated, Singapore.

3. Sebastian, C., Hack, E., and Patterson, E. (2012) An approach to the validation of computational solid mechanics models for strain analysis. J. Strain Anal. Eng. 48, 36-47.

4. Hills, R. G. and Trucano, T. G. (1999) Statistical validation of engineering and scientific models: background. Sandia National Laboratories, Albuquerque, NM, Report No.SAND99-1256.

5. Liu, Y., Chen, W., Arendt, P., and Huang, H. (2011) Toward a better understanding of model validation metrics. J. Mech. Des. 133, $1-13$.

6. Chen, W., Baghdasaryan, L., Buranathiti, T., and Cao, J. (2004) Model validation via uncertainty propagation and data transformations. AIAA J. 42, 1406-1415.

7. Oberkampf, W. L., and Barone, M. F. (2006) Measures of agreement between computation and experiment: validation metrics. J. Comput. Phys. 217, 5-36.

8. Thacker, B., Doebling, S., Hemez, F., Anderson, M., Pepin, J. and Rodriguez, E. (2004) Concepts of model verification and validation. Los Alamos National Laboratory. LA-14167-MS: 27.

9. Chianese, R. B., and Erdlac, R. J. (1988) The general solution to the distribution of stresses in a circular ring compressed by two forces acting along a diameter. Q. J. Mech. Appl. Math. 41, 239-247.

10. Ripperger, E. A., and Davids, N. (1947) Critical stresses in a circular ring. Proc. ASCE 112, 619-628.

11. Batista, M., and Usenik, J. (1996) Stresses in a circular ring under two forces acting along a diameter. J. Strain Anal. Eng. 31, 75-78.

12. Williamson, J. H. (1968) Least-squares fitting of a straight line. Can. J. Phys. 46, 1845-1847.

13. JCGM 101:2008. (2008) Evaluation of measurement data. Supplement 1 to the "Guide to the expression of uncertainty in measurement". Propagation of distributions using a Monte Carlo method. Joint Committee for Guides in Metrology.

14. ISO 13528:2005. Statistical methods for use in proficiency testing by interlaboratory comparisons.

15. Hesterberg, T. C. (1998) Simulation and bootstrapping for teaching statistics. Proc. American Statistical Association Proceedings of the Section on Statistical Education, 44-52.
16. Efron, B. (1979) Bootstrap methods: another look at the jackknife. Ann. Stat. 7, 1-26.

17. Timoshenko, S. (1922) On the distribution of stresses in a circular ring compressed by two forces acting along a diameter. Philosophical Magazine Series 6. 44, 1014-1019.

18. Timoshenko, S. (1951) Theory of Elasticity. McGraw-Hill, United States.

19. Timoshenko, S. (1910) Stresses in a circular ring compressed by two opposing forces. Procedings Kiev Polytechnic Institute. 9, 21-37.

20. Carl, W. N. (1939) Stresses and displacements in a hollow circular cylinder. Thesis, University of Michigan.

21. Vishay Micro-Measurements. (2010) The three-wire quarterbridge circuit. 11092, 221-223.

22. Watson, R. B. (2008) Bonded electrical resistance strain gages. In: Springer Handbook of Experimental Solid Mechanics. Springer, United States.

23. Vishay Micro-Measurements. (2008) Strain gage rosettes: selection, application and data reduction. 11065, 151-161.

24. JCGM 100:2008. (2008) Evaluation of measurement data Guide to the expression of the uncertainty in measurement. Joint Committee for Guides in Metrology.

25. Cantrell, C. A. (2008) Technical note: review of methods for linear least-squares fitting of data and application to atmospheric chemistry problems. Atmos. Chem. Phys. 8, 5477-5487.

26. Montero, W., Farag, R., Díaz, V., Ramirez, M., and Boada, B. (2011) Uncertainties associated with strain-measuring systems using resistance strain gauges. J. Strain Anal. Eng. 46, 1-13.

27. EA-4/02 M: 2013. (2013) Evaluation of the uncertainty of measurement in calibration.

28. ASTM E8M-01 (2001) Standard Test Methods for Tension Testing of Metallic Materials [Metric]. ASTM International, West Conshohocken, PA.

29. ASTM E111 - 04 (2004) Standard Test Method for Young's Modulus, Tangent Modulus, and Chord Modulus. ASTM International, West Conshohocken, PA.

30. ASTM E132-04 (2010) Standard Test Method for Poisson's Ratio at Room Temperature. ASTM International, West Conshohocken, PA.

31. Gabauer, W. (2000) Manual of codes of practice for the determination of uncertainties in mechanical tests on metallic materials, the determination of uncertainties in tensile testing. Project No. SMT4-CT97-2165, UNCERT COP. 7.

32. Adèr, M., Adèr, H. J., and Mellenbergh, G. (2008) Advising on Research Methods: A consultant's Companion. Johannes van Kessel Publishing, Netherlands.

33. Carpenter, J., and Bithell, J. (2000) Bootstrap confidence intervals: when, which, what? A practical guide for medical statisticians. Stat. Med. 19, 1141-1164.

34. Hesterberg, T. (2014) What teachers should know about the bootstrap: resampling in the undergraduate statistics curriculum. arXiv preprint arXiv:1411.5279.

35. Schwer, L. E. (2007) An overview of the PTC 60/V\&V 10: guide for verification and validation in computational solid mechanics. Eng. Comput. 23, 245-252. 